\title{
Tumorektomie oder Mastektomie?
}

\author{
Wahl des Operationsverfahrens für Stadien $T_{1}-T_{3} N_{x} M_{0}$ des Mammakarzinoms
}

J. Benz ${ }^{1}$, G. Berclaz ${ }^{1}$, V. Dupont Lampert ${ }^{2}$, E. Eicher ${ }^{3}$, F. Harder ${ }^{2}$, O. Köchli ${ }^{1}$, U. T. Laffer ${ }^{2}$, J. G. Poell ${ }^{4}$, M. Trutmann ${ }^{5}$, M. Zuber ${ }^{2}$

Arbeitsgruppe «Guideline Mammakarzinom, Chirurgische Therapie»

1 Schweizerische Gesellschaft für Gynäkologie und Geburtshilfe

2 Schweizerische Gesellschaft für Chirurgie

3 FMH (Leitung)

4 Schweizerische Gesellschaft für Plastische, Rekonstruktive und Ästhetische Chirurgie

5 Schweizerische Ärztezeitung

Korrespondenz:

Prof. Dr. med. U. T. Laffer

Spitalzentrum Biel

Chirurgische Klinik

CH-2501 Biel

E-Mail: laffer@gmx.ch

\section{Kurzfassung}

1. Patientinnen mit einem Mammakarzinom ist grundsätzlich die brusterhaltende Chirurgie gefolgt von Radiotherapie zu empfehlen ausser den unter 2. erwähnten Kontraindikationen

2. Kontraindikationen der brusterhaltenden Therapie sind:

- Multizentrizität des Tumors, d.h. Tumor in mehreren Quadranten;

- tumorbefallene Schnittränder trotz Nachresektionen;

- nach Tumorektomie, sofern nach Reexzision im gleichen Eingriff eine kosmetisch akzeptable Brusterhaltung nicht möglich ist;

- voraussehbar unbefriedigendes kosmetisches Resultat als Folge eines ungünstigen Verhältnisses zwischen Tumorgrösse und Brustvolumen;

- die postoperative Radiotherapie wird von der Patientin abgelehnt oder ist nach Rücksprache mit dem Radioonkologen nicht möglich;

- der klare Wunsch der Patientin nach Mastektomie;

- Rezidiv in bestrahlter Brust.

3. Keine Kontraindikation für eine brusterhaltende Therapie sind:

- zentral liegende (retromamilläre) Tumoren, z. B. M. Paget;

- Tumorbefall der axillären Lymphknoten;

- Brustimplantate;

- extensive intraduktale Komponente (EIC);

- Tumorgrösse;

- höheres Alter der Patientin.

4. Folgende Empfehlungen sind für ein optimales klinisches und kosmetisches Resultat zu beachten:

- nicht palpable Karzinome müssen präoperativ lokalisiert werden.

- die Schnittführung erfolgt über dem Tumor.

- radiäre Inzisionen der Haut sollten möglichst vermieden werden.

- Die Inzisionen zur Entfernung des Primärtumors und für die Axilladissektion sollten durch getrennte Zugänge durchgeführt werden, ausser wenn beide Schnittführungen anatomisch übereinstimmen, kosmetisch vertretbar sind und eine übersichtliche Operation beider Strukturen (Primärtumor und Axilla) ermöglichen

- Die Entfernung des Brustdrüsengewebes mit dem Tumor erfolgt radiär entsprechend dem Verlauf der Milchgänge in einem einzigen Gewebeblock.

- Um die topographische Orientierung für den Pathologen (und den Operateur) zu erleichtern, muss das Exzisat mit Fäden oder Clips markiert werden. Eine Entfernung der Haut ist, ausser bei Verdacht auf Infiltration, nicht notwendig. 
- Tumorbefallene Schnittränder müssen nachreseziert werden.

- Bei nicht palpablen, kleinen Läsionen muss intraoperativ mittels Präparateradiographie der Nachweis erbracht werden, dass die Läsion vollständig entfernt worden ist.

5. Eine Mastektomie kommt in Frage, wenn eine brusterhaltende Therapie nicht möglich oder unerwünscht ist.

Eine einfache Mastektomie (ohne Axilladissektion) kommt nur in Frage, wenn das histologische Staging der Axilla keine therapeutischen Konsequenzen hat und wenn klinisch kein Verdacht auf Tumorbefall der axillären Lymphknoten besteht.

Patientinnen sollten vor der Mastektomie auf die Rekonstruktionsmöglichkeiten aufmerksam gemacht werden. Die Rekonstruktionsmöglichkeiten sollten mit der Patientin besprochen werden.

6. Die objektive Aufklärung der Patientin über die Vor- und Nachteile aller Methoden durch den behandelnden Arzt muss dem Entscheid zwischen Brusterhaltung oder Mastektomie vorausgehen.

Die Körperform wird durch die brusterhaltende Therapie weniger gestört als durch die Mastektomie, und weniger Frauen empfinden körperliche und sexuelle Veränderungen.

Es gibt jedoch keine Evidenz, dass sich die psychologische Morbidität der Frauen oder ihre Gesamtlebensqualität nach brusterhaltender Therapie oder Mastektomie unterscheiden.

Gewöhnlich genügt eine einzelne Besprechung nicht, um die für eine Entscheidung notwendigen Informationen zu verarbeiten.

Es sollte der Patientin zugestanden werden, eine zweite Meinung einzuholen.

\section{Einleitung}

Bis Mitte der 80er Jahre bestand die operative Behandlung des Mammakarzinoms in der Entfernung der Brust in Form einer totalen Mastektomie mit der Entfernung der axillären Lymphknoten. Seither konnte in randomisierten Studien nachgewiesen werden, dass das Gesamtüberleben nach brusterhaltender Therapie (Entfernung des Tumors unter Belassung des gesunden Brustgewebes und anschliessende Radiotherapie) im Vergleich zur Mastektomie identisch ist (vgl. Tab. 1).

Unter Beachtung gewisser Kontraindikationen hängt die Wahl zwischen brusterhaltender Therapie und Mastektomie (und eventuell Rekonstruktion) von individuellen Umständen und persönlichen Wünschen ab.

\section{Zielsetzung}

Diese Guideline berücksichtigt für beide Operationsverfahren Evidenzen sowohl für das rezidiv- freie Überleben wie auch für das Gesamtüberleben. Die Guideline soll Ärztinnen und Ärzten sowie Patientinnen helfen, den Entscheid für die klinisch effizienteste und persönlich akzeptabelste chirurgische Behandlung unter Berücksichtigung der Lebensqualität zu treffen.

\section{Definitionen}

Die brusterhaltende Therapie besteht in der Entfernung des Tumors umgeben mit gesundem Gewebe, gefolgt von Radiotherapie, mit dem Ziel einer kosmetisch zufriedenstellenden Brust.

Die Mastektomie besteht in der vollständigen Entfernung des gesamten Brustdrüsengewebes inkl. Mamille und Areola.

Die Beurteilung des axillären Lymphknotenstatus ist grundsätzlich Bestandteil beider Verfahren.

Der Einsatz systemischer Behandlungsverfahren (Einfluss auf Hormon- oder Chemotherapie) ist bei beiden Optionen derselbe. 


\section{Grade der Evidenz}

Grad I: Die Evidenz ist aufgrund randomisierter kontrollierter Studien (oder Meta-Analysen) von genügendem Umfang derart, dass die Gefahr, dass sie falsch positive oder falsch negative Resultate beinhalten, gering ist.

Grad II: Die Evidenz basiert auf randomisierten kontrollierten Studien, welche jedoch zu klein sind, um ihnen Grad I zuzusprechen; sie können positive Trends, welche jedoch nicht signifikant sind, oder gar keine Trends zeigen. Sie sind mit einem hohen Risiko falsch negativer Resultate verbunden.

Grad III: Die Evidenz basiert auf nicht randomisierten Kontroll- oder Kohortenstudien, Fallserien, Fallkontrollstudien oder Querschnittsstudien (die beiden letzteren Studientypen wurden nicht berücksichtigt).

Grad IV: Die Evidenz beruht auf der Meinung angesehender ExpertInnen oder Expertengremien, wie sie in publizierten Konsenskonferenzen oder in Guidelines angegeben werden.

Grad V: Die Evidenz basiert auf der Meinung derjenigen Personen, welche diese Guidelines geschrieben oder aktualisiert haben, beruhend auf ihrer Erfahrung, ihrer Kenntnis der einschlägigen Literatur und der Diskussion mit ihren Fachkollegen.

Die Evidenzgrade der einzelnen Aussagen sind mit $\varepsilon_{1}, \varepsilon_{2}, \varepsilon_{3}$ und $\varepsilon_{4}$ gekennzeichnet. Evidenzgrad V entspricht dem Konsens der Arbeitsgruppe und wird nicht speziell vermerkt. wurde, dass die Daten durch eine der teilnehmenden Kliniken nicht korrekt erfasst worden waren. Eine erneute Auswertung der Resultate ohne die Daten dieses Zentrums änderte jedoch nichts an der Schlussfolgerung, dass beide Verfahren gleichwertig sind $[3,4,9,10]$.

Das Risiko für ein Lokalrezidiv nach brusterhaltender Therapie beträgt 1-2\% pro Jahr; es ist abhängig von der chirurgischen Radikalität, vom Alter der Patientin, vom histologischen Tumorstadium und von der adjuvanten Therapie. Es ist vergleichbar mit dem Risiko eines Karzinoms in der kontralateralen Brust $\left(\varepsilon_{3}\right)$ [11]. Auch nach einer radikalen Mastektomie besteht das Risiko eines lokalen Brustwandrezidives $[5,12,13]$.

Adjuvante Chemo-/Hormontherapien haben zudem zu einer weiteren Senkung der Lokalrezidivrate geführt [14, 15: $\left.\varepsilon_{1} ; 16: \varepsilon_{3}, 17\right]$.

Basierend auf dieser Datenlage kann die Wahl zwischen den beiden Verfahren aufgrund anderer Überlegungen getroffen werden. Der Vorteil der brusterhaltenden Therapie ist die Konservierung der Brust, der Nachteil ist die Notwendigkeit der Bestrahlung und die aufwendige Nachsorge. Die Bestrahlung ist zeitintensiv und kann auch logistisch schwierig sein. Zudem kann sie Nebenwirkungen verursachen [18, 19: $\left.\varepsilon_{3}\right]$.

Die brusterhaltende Therapie stellt hohe Anforderungen an das gesamte Behandlungsteam sowohl in der primären Behandlung wie in der Nachsorge.

\section{Kontraindikationen der brusterhaltenden Therapie} haltende Chirurgie gefolgt von Radiotherapie zu empfehlen ausser den unter 2. erwähnten Fällen.

Sechs randomisierte und kontrollierte Studien haben gezeigt, dass bei Patientinnen mit operablem Mammakarzinom die Ergebnisse in bezug auf rezidivfreies Überleben und Gesamtüberleben nach brusterhaltender Chirurgie, gefolgt von Radiotherapie, und nach Mastektomie, in der Regel ohne Radiotherapie, gleichwertig sind [1-9: $\varepsilon_{1}$ ] (Tab. 1); vgl. auch Guideline «Radiotherapie».

Die multizentrische NSABP-Studie B-06 mit 1843 Mammakarzinompatientinnen hat die statistisch höchste Aussagekraft. Nach einer medianen Beobachtungszeit von 12 Jahren waren das rezidivfreie Überleben und das Gesamtüberleben bei allen Patientinnen identisch, unabhängig davon, ob eine brusterhaltende Therapie mit Radiotherapie oder eine Mastektomie durchgeführt wurde. Das Rezidiv in der erhaltenen Brust war statistisch signifikant häufiger, wenn keine Radiotherapie erfolgte [4: $\varepsilon_{1}$ ]. Die Resultate der NSABP-Studie B-06 wurden in Frage gestellt, nachdem bekannt
2. Kontraindikationen der brusterhaltenden Therapie sind:

- Multizentrizität des Tumors, d.h. Tumoren in mehreren Quadranten [20].

- Tumorbefallene Schnittränder trotz Nachresektionen.

- Nach Tumorektomie, sofern nach Reexzision im gleichen Eingriff eine kosmetisch akzeptable Brusterhaltung nicht möglich ist.

- Voraussehbar unbefriedigendes kosmetisches Resultat als Folge eines ungünstigen Verhältnisses zwischen Tumorgrösse und Brustvolumen.

- Die postoperative Radiotherapie wird von der Patientin abgelehnt oder ist nach Rücksprache mit dem Radioonkologen nicht möglich.

- Der klare Wunsch der Patientin nach Mastektomie.

- Rezidiv in bestrahlter Brust. 
Tumorbefallene Resektionsränder bedeuten eine erhöhte Lokalrezidivrate trotz Nachbestrahlung [21: $\left.\varepsilon_{3}\right]$. Bei histologisch gesichertem Tumorbefall der Resektionsränder müssen eine Nachresektion oder eine Mastektomie durchgeführt werden.

Die Tumorgrösse korreliert in einigen, aber nicht in allen Untersuchungen mit dem Risiko eines Rezidivs nach brusterhaltender Therapie [1, 5, 22-24].

Die Behandlung des duktalen Carcinoma in situ wird in einem folgenden Kapitel dieser Guideline diskutiert.

Kontraindikationen für die Radiotherapie

Es gibt körperliche Gebrechen, welche den Einsatz der Radiotherapie erschweren. Eine weitere Kontraindikation kann eine vorgängige therapeutische Bestrahlung der Brust sein. In diesen Fällen empfiehlt sich eine präoperative Rücksprache mit dem Radioonkologen. Es gibt auch relative Kontraindikationen, wie Kollagenosen in der Anamnese.

\section{Besondere Situationen}

3. Keine Kontraindikation für eine brust-
erhaltende Therapie sind:
- zentral liegende (retromamilläre)
Tumoren (z. B. M. Paget);
- Tumorbefall der axillären Lymphknoten;
- Brustimplantate;
- extensive intraduktale Komponente
(EIC);
- Tumorgrösse;
- höheres Alter der Patientin.

Zentral liegende (retromamilläre) Tumoren

Die zentral liegenden (retromamillären) Tumoren zeigen die gleiche Gesamtprognose wie laterale Läsionen und sind keine Kontraindikation für eine brusterhaltende Therapie [25: $\varepsilon_{3}$ ]. Diese Läsionen sollten auf die gleiche Art und Weise wie die peripheren Läsionen entfernt werden. Dies bedeutet, falls notwendig, die Entfernung des gesamten Mamillen-Areola-Komplexes. Das erste Ziel ist dabei ebenfalls das Erreichen von tumorfreien Schnitträndern. Der MamillenAreola-Komplex kann plastisch-chirurgisch, eventuell kombiniert mit Tätowierung, rekonstruiert werden $\left(\varepsilon_{5}\right)$.

\section{Befall der axillären Lymphknoten}

Eine nodal-positive Axilla ist keine Kontraindikation für eine brusterhaltende Therapie [4: $\left.\varepsilon_{1}\right]$.

\section{Brustimplantate}

Das Vorhandensein von Prothesen ist keine Kontraindikation für eine brusterhaltende Therapie, vorausgesetzt, dass der Tumor mit tumorfreien Schnitträndern entfernt werden kann. Die Prothese ist keine Kontraindikation für eine Radiotherapie [26]. Die Radiotherapie erhöht aber das Risiko für eine Kapselfibrose [27: $\varepsilon_{4}$ ].

\section{Extensive intraduktale Komponente}

Einige Autoren berichten, dass mikroskopische Merkmale, wie eine ausgedehnte intraduktale Komponente (EIC) des Tumors, mit einer erhöhten Wahrscheinlichkeit von Lokalrezidiven verbunden sind [1, 21, 22, 28-30: $\left.\varepsilon_{3}\right]$.

In Serien, in welchen die Resektionsränder gut kontrolliert wurden, zeigt sich, dass die extensive intraduktale Komponente nicht unbedingt eine Erhöhung des Lokalrezidives vorhersagt, vorausgesetzt, dass die Exzision im Gesunden erfolgt $\left[9,28,29,31,32,33: \varepsilon_{3}\right]$.

Bei der extensiven intraduktalen Komponente ist jedoch häufig ein positiver Schnittrand zu beobachten [34].

\section{Tumorgrösse}

Das kosmetische Resultat der brusterhaltenden Therapie ist abhängig vom Volumenverhältnis zwischen entferntem Brustgewebe und Gesamtbrust und nicht alleine vom absoluten Volumen des entfernten Gewebes. Selten einmal kann ein Tumor in einer kleinen Brust so gross sein, dass ein akzeptables kosmetisches Resultat nach brusterhaltender Therapie nicht erreicht werden kann. In diesen Fällen sind eine onkoplastische Defektdeckung nach partieller oder totaler Mastektomie und häufig eine Anpassung der Gegenseite notwendig.

Höheres Alter der Patientin

Auch bei Patientinnen im fortgeschrittenen Alter kann die brusterhaltende Therapie angeboten werden.

\section{Empfehlungen für die chirurgische Technik der Tumorektomie}

Die Beschreibung der Technik der brusterhaltenden Therapie ist aufgrund verschiedener Expertenerfahrungen erarbeitet worden. 
Abbildung 1

Die Standardinzisionen sind bogenförmig direkt über den Tumor anzulegen (a).

Radiäre Inzisionen sind zu meiden (b). Nach [86].

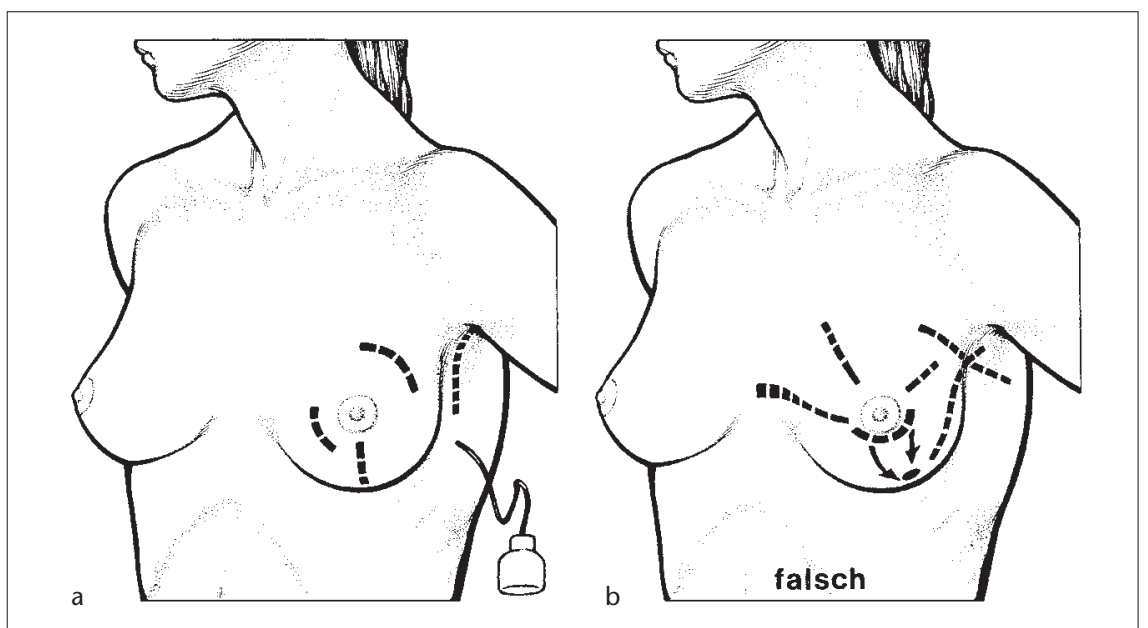

4. Folgende Empfehlungen sind für ein optimales klinisches und kosmetisches Resultat zu beachten:

- Nicht palpable Karzinome müssen präoperativ lokalisiert werden.1

- Die Schnittführung erfolgt über dem Tumor.

- Radiäre Inzisionen der Haut sollten möglichst vermieden werden.

- Die Inzisionen zur Entfernung des Primärtumors und für die Axilladissektion sollten durch getrennte Zugänge durchgeführt werden, ausser wenn beide Schnittführungen anatomisch übereinstimmen, kosmetisch vertretbar sind und eine übersichtliche Operation beider Strukturen (Primärtumor und Axilla) ermöglichen.

- Die Entfernung des Brustdrüsengewebes mit dem Tumor erfolgt radiär entsprechend dem Verlauf der Milchgänge in einem einzigen Gewebeblock.

- Um die topographische Orientierung für den Pathologen (und den Operateur) zu erleichtern, muss das Exzisat mit Fäden oder Clips markiert werden. Eine Entfernung der Haut ist, ausser bei Verdacht auf Infiltration, nicht notwendig.

- Tumorbefallene Schnittränder müssen nachreseziert werden.

- Bei nicht palpablen, kleinen Läsionen muss intraoperativ mittels Präparatradiographie der Nachweis erbracht werden, dass die Läsion vollständig entfernt worden ist.
Nicht palpable Karzinome müssen präoperativ lokalisiert werden.

Die Lokalisation erfolgt mit präoperativer Mammographie oder Ultrasonographie; stereotaktische Verfahren sind erstrebenswert.

\section{Schnittführung der Haut (Abb. 1)}

Die Inzision sollte direkt über den Tumor gelegt werden. In der Regel sollten Inzisionen das Radiotherapiefeld nicht überschreiten (Mitte Sternum bis vordere Axillarlinie). Eine Tunnelierung, welche von einer peri-areolären Inzision ausgeht und zu einem mehr peripher gelegenen Tumor führt, ist unübersichtlich, erhöht die Gefahr einer unvollständigen Resektion und ist deshalb zu vermeiden. Nachresektion und spätere Beurteilung der operierten Brust sind nach einem solchen Vorgehen zusätzlich erschwert.

An der Brust geben radiäre Inzisionen eher schlechtere kosmetische Resultate ausser in der 6-Uhr-Achse. Eine radiäre Schnittführung über dem Tumor, welche in die Axilla erweitert wird, führt zu einer Asymmetrie der Brust.

\section{Schnittführung an der Brustdrüse}

Die Entfernung des tumorbefallenen Brustdrüsenkörpers sollte mit radiärer Schnittführung in Richtung der Milchgänge erfolgen. Insbesondere wird damit die Rekonstruktion der Brustdrüse wesentlich erleichtert.

Die Exzision eines karzinomverdächtigen Befundes muss immer als Tumorektomie (excision en bloc) mit intaktem und von gesundem Gewebe umgebenem Präparat durchgeführt werden. Damit können viele dieser Tumoren bereits durch die erste Operation mit tumorfreien Schnitträndern (Schnellschnitt) entfernt werden, wodurch die Notwendigkeit einer zweiten Operation entfällt. Die Markierung des Tumorbettes mit Clips erfolgt zur Planung der Radiotherapie und zur Beurteilung der Mammographie im Rahmen der Nachsorge.

\section{Vorgehen bei tumorbefallenen \\ Exzisionsrändern}

Wenn die Exzisionsränder histologisch tumorbefallen sind, ist eine sofortige Nachresektion (Schnellschnitt) oder, wenn erst später erkannt, eine Nachresektion im Rahmen einer zweiten Operation (definitive Histologie) durchzuführen, und die Ränder sind neu zu beurteilen. Die Orientierung und Beurteilung im Zweiteingriff sind erschwert: Das beste Resultat kommt zustande, wenn bei der Primäroperation die vollständige Tumorentfernung und ein einwandfreies kosmetisches Ergebnis erzielt werden. Mit 
der Entfernung ausgedehnter Anteile des Brustgewebes, wie z.B. mit einer Quadrantektomie, kann die Rate von Lokalrezidiven wohl verringert werden, aber das kosmetische Resultat ist schlechter; Vorteile bezüglich Vermeidung von Metastasen oder Überleben werden damit nicht gewonnen [14, 35]. Ist eine Quadrantektomie unumgänglich, kann zur Verbesserung des kosmetischen Resultates eine onkoplastische Korrektur durchgeführt werden (Latissimus-dorsiLappen, allenfalls mit Mammareduktionsplastik der Gegenseite, Rotation der Drüsenkörper und Adaptation).

Ein Schnittrand wird als tumorfrei bezeichnet, wenn mikroskopisch keine malignen Zellen an der Schnittfläche gefunden werden. In der NSABP-B-06-Studie [4] wurden tumorfreie Schnittränder, aber kein minimaler tumorfreier Gewebsaum verlangt. Die Schnellschnittechnik erlaubt die primäre Evaluation an unsicheren Stellen, sie genügt jedoch nicht zur vollständigen Beurteilung der Schnittränder. Dies ist erst im Rahmen der definitiven histologischen Aufarbeitung des Präparates möglich.

Können keine tumorfreien Schnittränder erzielt werden, muss amputiert werden, gegebenenfalls mit gleichzeitiger Rekonstruktion oder mit Rekonstruktion im Intervall.

\section{Die Mastektomie}

5. Eine Mastektomie kommt in Frage, wenn eine brusterhaltende Therapie nicht möglich oder unerwünscht ist.

Eine einfache Mastektomie (ohne Axilladissektion) kommt nur in Frage, wenn das histologische Staging der Axilla keine therapeutischen Konsequenzen hat und wenn klinisch kein Verdacht auf Tumorbefall der axillären Lymphknoten besteht.

Patientinnen sollten vor der Mastektomie auf die Rekonstruktionsmöglichkeiten aufmerksam gemacht werden. Die Rekonstruktionsmöglichkeiten sollten mit der Patientin besprochen werden.

\section{Indikationen zur Mastektomie}

Vergleiche Kapitel «Kontraindikationen der brusterhaltenden Therapie»!

\section{Einleitung}

Die modifiziert radikale Mastektomie nach Patey/Dysin [36] ist die Standardtechnik bei der Mastektomie. Im Gegensatz zu diesen beiden
Autoren, welche noch eine Durchtrennung des M. pectoralis minor empfahlen, wird allerdings heute die Technik nach Auchincloss [37], ohne Durchtrennung des M. pectoralis minor, durchgeführt. Oberstes Ziel der Mastektomie ist eine vollständige Entfernung der Brustdrüse mit Brustwarze und Warzenhof unter Bildung von genügend grossen Hautlappen, um gute Voraussetzung für einen spannungsfreien Wundverschluss zu schaffen.

\section{Technik}

Die Hautinzision erfolgt elliptisch und horizontal mit Entfernung des gesamten Mamillen-AreolaKomplexes und unter Mitnahme eventueller Narben bei vorausgegangener Probeexzision. Die Hautinzision soll die laterale Begrenzung des Sternums nicht erreichen und nach lateral soweit ausgedehnt werden, dass eine gute Exploration der Axilla möglich ist. Eine Entfernung der Haut über dem Tumor bei exzentrischer Lage ist, falls klinisch nicht der Verdacht auf eine Infiltration besteht, nicht obligat.

Die gesamte Brustdrüse ist in toto zu entfernen. Die Dissektionsebene liegt zwischen der subkutan gelegenen Fettschicht und der Drüse, entlang einer ganz feinen, immer wieder unterbrochenen Faszie.

Die Wundhöhle wird drainiert, Kompressionsverbände sollten nicht angewendet werden [38].

\section{Komplikationen}

Die Mortalität liegt nach Mastektomie - auch bei über 75jährigen Patientinnen - weit unter $1 \%$ [39]. Die Infektrate wird in der Literatur zwischen $<5$ und $>15 \%$ beschrieben [39-41]. Sie ist erhöht bei zweizeitigen Operationen (nach vorausgehender Probeexzision) und wenn die Drainagen zu lange belassen werden.

Eine Antibiotikaprophylaxe führt zu einer statistisch signifikanten Senkung der Infektrate [42-45: $\varepsilon_{1}$ ]. Die Antibiotikaprophylaxe ist bei High-risk-Patientinnen zu empfehlen. Zu dieser Gruppe gehören: Patientinnen mit zweitzeitiger Operation, mit langer Operationsdauer und mit bestrahlter Brust sowie adipöse Patientinnen.

Die Lappennekrose ist selten, am ehesten bei zu dünnem Hautlappen, nach Hautverschluss unter Spannung und bei Kompressionsverbänden [46].

Das postoperative Wundserom nach Mastektomie ist häufig. Damit begründet ist auch die gute Drainage nach Mastektomie, die erst entfernt werden sollte, wenn weniger als $50 \mathrm{ml}$ pro Tag gefördert werden [47-50], spätestens jedoch nach 5 Tagen $\left(\varepsilon_{5}\right)$. Nach Entfernung der Drainage auftretende Wundserome sollten punktiert werden. 


\section{Die Rekonstruktion}

\section{Vorbesprechung}

Vor einer Mastektomie sollte die Patientin über alle rekonstruktiven Möglichkeiten informiert sein. Wesentlich ist die Information über die primäre oder sekundäre Rekonstruktion. Je nach Methode kann eine sekundäre Rekonstruktion vorteilhafter sein (z. B. Implantat und Nachbestrahlung). Meist ist aber die primäre Rekonstruktion technisch einfacher durchzuführen. Sie ist auch für die Patientin weniger belastend, da sie sich eine zweite Narkose und die psychisch oft deprimierende Zeit, nur mit einer Brust leben zu müssen, erspart.

\section{Schnittführung}

Die spindelförmige Hautresektion sollte so hautsparend wie möglich sein, ohne die onkochirurgischen Erfordernisse zu kompromittieren. Idealerweise sollte die Schnittführung mit dem plastischen Chirurgen vorbesprochen werden.

\section{Rekonstruktionsmethoden}

Autologe Rekonstruktion - Verschiedene Lappenplastiken werden verwendet und von verschiedenen Operateuren je nach eigenen Erfahrungen eingesetzt [51-53, 54-60, 61]. Die gebräuchlichsten sind die erweiterte Latissimus-dorsiund die TRAM-Plastik.

Das Volumen des Latissimus-dorsi-Lappens erlaubt die Rekonstruktion einer kleineren oder mittleren Brust [51, 52, 62, 60].

Der TRAM kann fast unbeschränkt gross gewählt werden.

Implantat-Ein Silikonimplantat kann primär oder nach Expansion der Haut sekundär, subkutan oder auch submuskulär eingesetzt werden. Früher oder später wird dieses in den meisten Fällen ersetzt werden müssen, im Gegensatz zur autologen Rekonstruktion, die meist endgültig ist. Die Konsistenz und das Verhalten einer implantatrekonstruierten Brust ist qualitativ immer schlechter als die nach einer gelungenen autologen Rekonstruktion [63-74].

Zusätzlich existieren technisch gemischte Rekonstruktionen.

\section{Patientinneninformation}

6. Die objektive Aufklärung der Patientin über die Vor- und Nachteile aller Methoden durch den behandelnden Arzt muss dem Entscheid zwischen Brusterhaltung oder Mastektomie vorausgehen.

Die Körperform wird durch die brusterhaltende Therapie weniger gestört als durch die Mastektomie [75-77], und weniger Frauen empfinden körperliche und sexuelle Veränderungen [75, 77]. Es gibt jedoch keine Evidenz, dass sich die psychologische Morbidität der Frauen oder ihre Gesamtlebensqualität nach brusterhaltender Therapie oder Mastektomie unterscheiden [76, 79].

Gewöhnlich genügt eine einzelne Besprechung nicht, um die für eine Entscheidung notwendigen Informationen zu verarbeiten.

Es sollte der Patientin zugestanden werden, eine zweite Meinung einzuholen.

In einer prospektiven nicht randomisierten Studie berichteten $65 \%$ von 269 Patientinnen mit einem Mammakarzinom Stadium pT1-pT3, dass die Krebsangst ihre Primärangst war und dass diese ausgeprägter war als die Angst vor dem Verlust der Brust.

Weiterhin konnte ein Jahr nach dem chirurgischen Eingriff bezüglich Krebsangst oder Depressionen kein Unterschied zwischen Frauen nach brusterhaltender Therapie und nach Mastektomie festgestellt werden.

Hingegen zeigten Patientinnen, denen der Operateur die Wahl zwischen den beiden Operationsmethoden überliess, weniger Depressionen als Patientinnen, die von anderen Operateuren behandelt wurden $(\mathrm{p}=0,06)$ [80: $\left.\varepsilon_{3}\right]$. Diese Studie lässt jedoch offen, ob die geringeren Depressionen durch die Möglichkeit der Wahl zwischen zwei Operationsmethoden oder durch die grössere Sympathie zum Chirurgen verursacht wurden.

Die Wichtigkeit der Kommunikation zwischen Arzt und Patientin in bezug auf die Therapieentscheidung und die psychologische Verarbeitung wird aber zunehmend anerkannt $[81,82]$. In der Tat konnte nach 6 Monaten eine Korrelation zwischen der psychologischen Verarbeitung der Patientin und der Wahrnehmung des Arztverhaltens während der Bekanntgabe der Diagnose nachgewiesen werden [82]. 
Vor allem sind Diskussionen mit der Patientin über die gesamte Problematik von grosser Bedeutung. Die Kommunikation ist erfolgreicher, wenn zusätzlich ein Familienmitglied anwesend ist und der Patientin eine schriftliche Zusammenfassung der Vor- und Nachteile der Behandlungsmöglichkeiten abgegeben wird, welche sie in Ruhe studieren kann [83].

Um die Informationen zu verarbeiten, muss der Patientin Zeit und - falls sie dies wünscht die Möglichkeit einer weiteren Besprechung gegeben werden. Um unnötige Ängste zu vermeiden, sollte der Patientin erklärt werden, dass ihre Heilungschancen nicht kompromittiert werden, wenn sie Zeit für eine Entscheidungsfindung braucht. Nach gegenwärtigem Wissensstand ist der Tumor bei Diagnosestellung vermutlich schon seit einigen Monaten oder Jahren im präklinischen Stadium vorhanden gewesen $\left(\boldsymbol{\varepsilon}_{5}\right)$.

Verschiedene Studien haben nachgewiesen, dass in solchen Gesprächen die Präferenzen (bias) des Operateurs deutlich zum Ausdruck kommen [80, 84, 85]. Obwohl es manchmal wichtig sein kann, der Patientin eine spezifische Empfehlung zu geben, sollten diese Empfehlungen neutral, ausgewogen und so detailliert begründet werden, wie es die Patientin wünscht.

\section{Literatur}

1 Arriagada R, Lê MG, Rochard F, Contesso G. Conservative treatment versus mastectomy in early breast cancer: patterns of failure with 15 years of follow-up data. Institut GustaveRoussy Breast Cancer Group. J Clin Oncol 1996;14:1558-64.

2 Blichert-Toft M, Rose C, Andersen JA Overgaard M, Axelsson CK, Andersen KW, et al. Danish randomized trial comparing breast conservation therapy with mastectomy: six years of life-table analysis. Danish Breast Cancer Cooperative Group. J Natl Cancer Inst Monogr 1992;11:19-25.

3 Fisher B, Redmond C, Poisson R, Margolese R, Wolmark N, Wickerham L, et al. Eight-year results of a randomized clinical trial comparing total mastectomy and lumpectomy with or without irradiation in the treatment of breast cancer. N Engl J Med 1989;320:822-8.

4 Fisher B, Anderson S, Redmond CK, Wolmark M, Wickerham DL, Cronin WM. Reanalysis and results after 12 years of follow-up in a randomized clinical trial comparing total mastectomy with lumpectomy with or without irradiation in the treatment of breast cancer. N Engl J Med $1995 ; 333: 1456-61$
5 van Dongen JA, Bartelink H, Fentiman IS, Lerut T, Mignolet F, Olthuis G, et al. Factors influencing local relapse and survival and results of salvage treatment after breast-conserving therapy in operable breast cancer: EORTC trial 10801, breast conservation compared with mastectomy in TNM stage I and II breast cancer. Eur J Cancer 1992;28A:801-5.

6 Veronesi U, Saccozzi R, Del Vecchio M, Banfi A, Clemente C, De Lena M, et al. Comparing radical mastectomy with quadrantectomy, axillary dissection, and radiotherapy in patients with small cancers of the breast. N Engl J Med 1981; 305:6-11.

7 Veronesi U, Banfi A, Salvadori B, Luini A, Saccozzi R, Zucali $\mathrm{R}$, et al. Breast conservation is the treatment of choice in small breast cancer: long-term results of a randomized trial. Eur J Cancer 1990; 26:668-70.

8 Jacobson JA, Danforth DN, Cowan KH, D'Angelo T, Steinberg S, Pierce L, et al. Ten-year results of a comparison of conservation with mastectomy in the treatment of stage I and II breast cancer. N Engl J Med 1995;332:907-11.

9 Fisher B, Bauer M, Margolese R, Poisson R, Pilch Y, Redmond $\mathrm{C}$, et al. Five-year results of a randomized clinical trial comparing total mastectomy and segmental mastectomy with or without radiation in the treatment of breast cancer. N Engl J Med 1985;312:665-73.

10 Christian MC, McCabe MS, Korn EL, Abrams JS, Kaplan RS, Friedman MA. The National Cancer Institute audit of the National Surgical Adjuvant Breast and Bowel Project Protocol B-06. N Engl J Med 1995;333:1469-74.

11 Kurtz JM. How to predict the risk of local relapse in the preserved breast. Recent Results Cancer Res 1996;140:263-72.

12 Hacene K, Le Doussal V, Rouesse J, Brunet M. Predicting distant metastases in operable breast cancer patients. Cancer 1990;66:2034-43.

13 Cody HS, Laughlin EH, Trillo C, Urban JA. Have changing treatment patterns affected outcome for operable breast cancer? Ten-year follow-up in 1288 patients, 1965-1978. Ann Surg 1991; 213:297-307.

14 Goldhirsch A, Glick JH, Gelber RD, Coates AS, Senn HJ. Meeting highlights: International Consensus Panel on the treatment of primary breast cancer. J Clin Oncol 2001;19:3817-27.

15 Early Breast Cancer Trialists' Collaborative Group. Polychemotherapy for early breast cancer: an overview of the randomised trials. Lancet 1998;352:930-42.

16 Fisher B, Dignam J, Mamounas EP, Costantino JP, Wickerham DL, Redmond C, et al. Sequential methotrexate and fluorouracil for the treatment of node-negative breast cancer patients with estro-gen receptor-negative tumors: eight-year results from National Surgical Adjuvant Breast and Bowel Project (NSABP) B-13 and first report of findings from NSABP B-19 comparing methotrexate and fluorouracil with conventional cyclophosphamide, methotrexate, and fluouracil. J Clin Oncol 1996;14:1982-92. 
17 Margolese R. Surgical considerations in selecting local therapy. J Natl Cancer Inst Monogr 1992; 11:41-8.

18 Bedwinek JM, Brady L, Perez CA, Goodman R, Kramer S, Grundy G. Irradiation as the primary management of stage I and II adenocarcinoma of the breast: analysis of the RTOG breast registry. Cancer Clin Trials 1980; 3:11-8.

19 Kantorowitz DA, Poulter CA, Rubin P, Patterson E, Sobel SH, Sischy B, et al. Treatment of breast cancer and segmental mastectomy alone or segmental mastectomy plus radiation. Radiother Oncol 1989;15:141-50.

20 Leopold KA, Recht A, Schnitt SJ, Connolly JL, Rose MA, Silver B, et al. Results of conservative surgery and radiation therapy for multiple synchronous cancers of the breast. Int J Radiat Oncol Biol Phys 1989;16:11-6.

21 Kurtz J, Jacquemier J, Amalric R, Brandone $\mathrm{H}$, Ayme Y, Hans D, et al. Why are local recurrences after breast conserving therapy more frequent in younger patients? J Clin Oncol 1990; 8:591-8.

22 Zafrani B, Vielh P, Fourquet A, Mosseri V, Durand JC, Salmon RJ, et al. Conservative treatment of early breast cancer: prognostic value of the ductal in situ component and other pathological variables on local control and survival. Long-term results. Eur J Cancer Clin Oncol $1989 ; 25: 1645-50$.

23 Chaudary MA, Hayward JL. Radiation therapy and limited surgery are not necessarily as good as modified radical mastectomy for the treatment of breast cancer. In: Wise L, Johnson H Jr, editors. Breast cancer: controversies in management. Armonk (NY): Futura Publishing; 1994. p. 147-64.

24 Eberlein TJ, Connolly JL, Schnitt SJ, Recht A, Osteen RT, Harris JR. Predictors of local recurrence following conservative breast surgery and radiation therapy. The influence of tumor size. Arch Surg 1990;125:771-7.

25 Fisher B, Redmond C, Fisher ER, Bauer M, Wolmark N, Wickerham L, et al. Ten-year results of a randomized clinical trial comparing radical mastectomy and total mastectomy with or without radiation. N Engl J Med 1985;312:674-81.

26 Ryu J, Yahalom J, Shank B, Chaglassian TA, McCormick B. Radiation therapy after breast augmentation or reconstruction in early or recurrent breast cancer. Cancer 1990;66:844-7.

27 Evans GR, Schusterman MA, Kroll SS, Miller MJ, Reece GP, Robb GL, et al. Reconstruction and the radiated breast: Is there a role for implants? Plast Reconstr Surg 1995;96:1111-5.

28 Clarke DH, Lê MG, Sarrazin D, Lacombe M-J, Fontaine F, Travagli J-P, et al. Analysis of localregional relapses in patients with early breast cancers treated by excision and radiotherapy: experience of the Institut Gustave-Roussy. Int J Radiat Oncol Biol Phys 1985;11:137-45.

29 Van Limbergen E, Van den Bogaert W, Van der Schueren E, Rijnders A. Tumor excision and radiotherapy as primary treatment of breast cancer: analysis of patient and treatment parameters and local control. Radiother Oncol 1987; 8:1-9.
30 Schnitt SJ, Connolly JL, Harris JR, Hellman S, Cohen RB. Pathological predictors of early local recurrence in stage I and II breast cancer treated by primary radiation therapy. Cancer 1984; 53:1049-57.

31 Fourquet A, Campana F, Zafrani B, Mosseri V, Vielh P, Durand JC, et al. Prognostic factors of breast recurrence in the conservative management of breast cancer: a 25-year follow-up. Int J Radiat Oncol Biol Phys 1989;17:719-25.

32 Fowble B, Solin L, Schultz D, Rubenstein J, Goodman R. Breast recurrence following conservative surgery and radiation: patterns of failure, prognosis, and pathologic findings from mastectomy specimens with implications for treatment. Int J Radiat Oncol Biol Phys 1990; 19:833-42.

33 Schnitt SJ, Abner A, Gelman R, Connolly JL, Recht A, Duda RB, et al. The relationship between microscopic margins of resection and the risk of local recurrence in patients with breast cancer treated with breast-conserving surgery and radiation therapy. Cancer1994; 74:1746-51.

34 Holland R, Connolly JL, Gelman R, Mravunac M, Hendriks J, Verbeek A, et al. The presence of an extensive intraductal component following a limited excision correlates with prominent residual disease in the remainder of the breast. J Clin Oncol 1990; 8:113-8.

35 Veronesi U, Volterrani F, Luini A, Saccozzi R, Del Vecchio M, Zucali R, et al. Quadrantectomy versus lumpectomy for small size breast cancer. Eur J Cancer 1990;26:671-3.

36 Patey D, Dysin W. The prognosis of carcinoma of the breast in relation to type of operation performed. Br J Cancer 1948;2:71.

37 Auchincloss H. Modified mastectomy. Am J Surg 1970;119:506

38 Morrow M, Harris JR. Local management of invasive breast cancer in diseases of the breast. In Harris JR, Lippman ME, Morrow M, Osborne CK (Editors). Second Edition Lippincott Williams \& Wilkins; 2000. p. 515-560.

39 Forouhi P, Dixon JM, Leonard RC, Chetty U. Prospective randomized study of surgical morbidity following primary systemic therapy for breast cancer. Br J Surg 1995;82(1):79-82.

40 Lipshy KA, Neifeld JP, Boyle RM, Frable WJ, Ronan S, Lotfi P, Bear HD, Horsley JS 3rd, Lawrence W Jr. Complications of mastectomy and their relationship to biopsy technique. Ann Surg Oncol 1996;3(3):290-4.

41 Bertin ML, Crowe J, Gordon SM., Determinants of surgical site infection after breast surgery. Am J Infect Control 1998;26(1):61-5.

42 Platt R, Zucker J, Zaleznik D, et al. Perioperative antibiotic prophylaxis and wound infection following breast surgery. J Antimicrob Chemother 1993;31:43.

43 Platt R, Zucker J, Zaleznik D, et al. Prophylaxis against wound infection following herniorrhaphy or breast surgery. J Antimicrob Chemother 1993; $31: 43$. 
44 Wagman LD, Tegtmeier B, Beatty JD, Kloth DD, Kokal WA, Riihimaki DU, Terz JJ. A prospective randomized double-blind study of the uses of antibiotics at the time of mastectomy. Surg Gynecol Obstet 1990;170(1):12-6.

45 Platt R, Zucker JR, Zaleznik DF, Hopkins CC, Dellinger EP, Karchmer AW, et al. Prophylaxis against wound infection following herniorrhaphy or breast surgery. J Infect Dis 1992;166(3):556-60.

46 Chilson T, Chan F, Lonser R, Wu TM, Aitken DR. Seroma prevention after modified radical mastectomy. Am Surg 1992;58:750.

47 Petrek JA, Peters M, Noris S, et al. Axillary lymphadenopathy: a prospective randomized trial of thirteen factors influencing drainage, including early or delayed arm mobilization. Arch Surg 1991;125:378.

48 Vinton AL, Traverso LW, Jolly PC. Wound complications after modified mastectomy compared with tylectomy with axillary lymph node dissection. Am J Surg 1991;161(5):584-8.

49 Oertli D, Laffer U, Haberthuer F, Kreuter U, Harder F. Perioperative and postoperative tranexamic acid reduces the local wound complication rate after surgery for breast cancer. Br J Surg 1994;81(6):856-9.

50 Rice DC, Morris SM, Sarr MG, Farnell MB, van Heerden JA, Grant CS, et al. Intraoperative topical tertracycline sclerotherapy following mastectomy: a prospective, randomized trial. J Surg Oncol 2000;73(4):224-7.

51 Bostwick J III. Plastic an reconstructive breast surgery. 2nd edition. St. Louis: Quality Medical Publishing; 2000.

52 Hartrampf R Jr. Breast reconstruction with living tissue. Philadelphia: Lippincott Williams \& Wilkins; 1991.

53 Allen R, Guarda H, Wall F, Dupin C, Glass C. Free flap breast reconstruction: the LSU experience (1984-1996). J La State Med Soc 1997;149:386-92.

54 Paige KT, Bostwick J, Trimbel Bried J, Jones G. A comparison of morbidity from bilateral unipedicled and unilateral, unipedicled TRAM flap reconstructions. Plast Reconstruct Surg 1998; 101:1819-27.

55 Biondeel PN, Vanderstraeten GG, Monstrey SJ, Van Landuyt K, Tonnard P, Lysens R, Matton G. The donor site morbidity of free DIEP flaps and free TRAM flaps for breast reconstruction. Br J Plast Surg 1097;50:322-30.

56 Blondeel PN. The sensate free superior gluteal artery perforator (S-GAP) flap: a valuable alternative in autologous breast reconstruction. Br J Plast Surg 1999;52: 185-93.

57 Blondeel PN, Demuynek M, Mete D, Monstrey SJ, Van Landuyt K, Matton G, Vanderstraeten GG. Sensory nerve repair in perforator flaps for autologous breast reconstruction: sensational or senseless? Br J Plast Surg 1999;52:37-44.

58 Schrudde J. Mammarekonstruktionen mittels Dermofettlappen. Handchirurgie, Mikrochirurgie, Plastische Chirurgie 1986;18:257-63.
59 Holle J, Pierini A. Breast reconstruction with an external oblique abdominis muscle turnover flap and bipedicled abdominal skin flap. Plast Reconstr Surg 1984;73:469-73.

60 Delay E, Gratadour AC, Jorquera F, Zlatoff P, Bremond A. Immediate autologous latissimus breast reconstruction after skin sparing mastectomy. Eur J Plast Surg 1999;22:111-8.

61 Spear SL. Surgery of the breast. Principles and art. Lippincott - Raven; 1998.

62 Kroll SS. Breast reconstruction with autologous tissue clinics in plastic surgery. Clin Plast Surg 1998;25:135-43.

63 Olbrisch RR, Miericke, B. Der Gewebe-Expander zum Brustwiederaufbau Erfahrungen und Ergebnisse mit mehr als 300 Expandern. Chirurg 1987;58:553-8.

64 Spear SL, Majidian A. Immediate breast reconstruction in two stages using textured, integrated-valve tissue expander and breast implants: a retrospective review of 171 consecutive breast reconstructions from 1989 to 1996. Plastic Reconstruct Surg 1998;101:53-63.

65 Gabriel SE, Woods JE, 0’Fallon M, Beard M, Kurland L, Melton J. Complications leading to surgery after breast implantation. N Engl J Med 1997;336: 677-82.

66 Beekman WH, Feitz R, Hage JJ, Mulder JW. Life span of silicone gel-filled mammary prostheses. Plastic Reconstruct Surgery 1997;100:1723-8.

67 Peters W, Smith D. Calcifieation of breast implant capsules: incidence, diagnosis and contributing factors. Ann Plastic Surg 1998;34:8-11.

68 Spear SL, Backer J. Classification of capsular contracture after prosthetic breast reconstruction. Plastic Reconstruct Surg 1995;96:1119-23.

69 Brown LS, Silverman BG, Berg WA. Rupture of silicone-gel breast implants: causes, sequelae, and diagnosis. Lancet 1997;350:1531-7.

70 Weizer G, Malone RS, Netscher DT, Walker LE, Thomby J. Utility of magnetic resonance imaging and ultrasonography in diagnosing breast implant rupture. Ann Plastic Surg 1995; 34:352-61.

71 Spiera Harry, Spiera R. Silicone breast implants and connective tissue disease: an overview. Mount Sinai J Med 1997;64:363-71.

72 Noone BR. A review of the possible health implications of silicone breast implants. Am Cancer Soc 1997;79:1747-56.

73 Edworthy SM, Martin L, Barr SG, Birdsell DC, Brand RE. A clinical study of the relationship between silicone breast implants and connective tissue disease. J Rheumatol 1998;25:254-60.

74 Clodius L. Silicones in plastic surgery - the situation today. Eur J Plast Surg 1999;22:55-7.

75 Beckmann J, Johansen L, Richardt C, Blichert-Toft M. Psychological reactions in younger women operated on for breast cancer. Amputation versus resection of the breast with special reference to body-image, sexual identity and sexual function. Dan Med Bull 1983; 30(Suppl 2):10-3. 
76 Ganz PA, Schag CC, Polinsky ML, Heinrich RL, Flack VF. Rehabilitation needs and breast cancer: the first month after primary therapy. Breast Cancer Res Treat 1987;10:243-53.

77 Schain WS, d'Angelo TM, Dunn ME, Lichter AS, Pierce LJ. Mastectomy versus conservative surgery and radiation therapy. Psychosocial consequences. Cancer 1994;73:1221-8.

78 De Haes JCJM, van Oostrom MA, Welvaart K. The effect of radical and conserving surgery on the quality of life of early breast cancer patients. J Eur Surg Oncol 1986;12:337-42.

79 Sacks NPM, Baum M. Primary management of carcinoma of the breast. Lancet 1993;342:1402-8.

80 Fallowfield LJ, Hall A, Maguire GP, Baum M. Psychological outcomes of different treatment policies in women with early breast cancer outside a clinical trial. Br Med J 1990;301:575-80.

81 Lerman C, Daly M, Walsh WP, Resch N, Seay J, Barsevick A, et al. Communications between patients with breast cancer and health care providers. Cancer 1993;72:2612-20.
82 Roberts CS, Cox CE, Reintgen DS, Baile WF Gibertini M. Influence of physician communication on newly diagnosed breast patients' psychologic adjustment and decision-making. Cancer 1994;74:336-41.

83 The Steering Committee on Clinical Practice Guidelines (Chair: McGregor M). Clinical practice guidelines for the care and treatment of breast cancer: a Canadian consensus document (consumer version). Ottawa: Canadian Medical Association; 1997. Obtainable from: Publications, Health Canada, Tunney's Pasture, Ottawa ON K1A 0K9.

84 Margolese RG. Breast cancer surgery: Who chooses and how? CMAJ 1994;150:331-3.

85 Tarbox BB, Rockwood JK, Abernathy CM. Are modified radical mastectomies done for T1 breast cancers because of surgeon's advice or patient's choice? Am J Surg 1992;164:417-22.

86 Harder F, Laffer U, Almendral AC, Hünig R, Torhorst JKH. Methodenwahl beim kleinen Mammacarcinom. Chirurg 1991;62:85-90.

Tabelle 1

Summary of survival and recurrence in prospective randomized trials of mastectomy versus breast-conserving surgery.

\begin{tabular}{|c|c|c|c|c|c|c|}
\hline Study group & Interventions & No. of patients & Follow-up & Survival & $\begin{array}{l}\text { Disease-free } \\
\text { survival }\end{array}$ & $\begin{array}{l}\text { Local } \\
\text { recurrence }\end{array}$ \\
\hline & & & year & $\%$ & $\%$ & $\%$ \\
\hline \multirow[t]{2}{*}{ Veronesi et al. (Milan) [7] } & $\mathrm{BCS}+\mathrm{RT}$ & 352 & 15 & 68 & - & 3,3 \\
\hline & TM & 349 & 15 & 66 & - & 2,3 \\
\hline \multirow[t]{3}{*}{ Fisher et al. (NSABP) [3] } & BCS & 636 & 8 & 83 & 64 & 39 \\
\hline & $\mathrm{BCS}+\mathrm{RT}$ & 629 & 8 & 84 & 71 & 10 \\
\hline & $\mathrm{TM}$ & 590 & 8 & 82 & 67 & 8 \\
\hline \multirow[t]{2}{*}{ Blichert-Toft et al. (Denmark) [2] } & $\mathrm{BCS}+\mathrm{RT}$ & 430 & 6 & 79 & 70 & - \\
\hline & TM & 429 & 6 & 82 & 66 & - \\
\hline \multirow[t]{2}{*}{ van Dongen et al. (EORTC) [5] } & $\mathrm{BCS}+\mathrm{RT}$ & 426 & 8 & 60 & - & 11 \\
\hline & TM & 456 & 8 & 60 & - & 8 \\
\hline \multirow[t]{2}{*}{ Jacobson et al. (NCI) [8] } & $\mathrm{BCS}+\mathrm{RT}$ & 121 & 10 & 77 & 72 & 5 \\
\hline & TM & 116 & 10 & 75 & 69 & 10 \\
\hline \multirow[t]{2}{*}{$\begin{array}{l}\text { Arriagada et al. } \\
\text { (Institut Gustave-Roussy) [1] }\end{array}$} & $\mathrm{BCS}+\mathrm{RT}$ & 88 & 15 & 73 & 55 & 9 \\
\hline & TM & 91 & 15 & 65 & 44 & 14 \\
\hline
\end{tabular}

NSABP: National Surgical Adjuvant Breast Project

$\mathrm{NCl}$ : National Cancer Institute

EORTC: European Organization for the Research and Treatment of Cancer

BCS: breast-conserving surgery

RT: radiotherapy

TM: total mastectomy 